Original Articles

\author{
Review Articles
}

Bone-Targeted Targeted Therapy in Metastatic Breast Cancer All Well-Established Knowledge?

Gampenrieder, S.; Rinnerthaler, G.; Greil, R. (Salzburg)

Side-Effects of Bone-Targeted Therapies in Advanced Breast Cancer Domschke, C.; Schütz, F. (Heidelberg)

Can Steroids Plus Surgery Become First Line Treatment of Idiopathic Granulomatous Mastitis?

Karanlik, H. (Istanbul); Ozgur, I. (Van); Simsek, S.; Fathalizadeh, A.; Serin, K.; Dursun, M.; Kurul, S. (Istanbul)

Taxanes plus Trastuzumab Compared to Oral Vinorelbine plus Trastuzumab in HER2-Overexpressing Metastatic Breast Cancer

Bergen, E.; Berghoff, A.; Rudas, M.; Dubsky, P.; De Vries, C. (Vienna); Sattlberger, C. (Vöcklabruck); Mader, R.; Zagouri, F.; Sparber, C.; Fitzal, F.; Gnant, M.; Rottenfusser, A.; Zielinski, C.; Preusser, M.; Steger, G.; Bartsch, R. (Vienna)

Guideline Conformity Treatment in Young Women with Early-Onset Breast Cancer in Germany

Pursche, T.; Hedderich, M.; Heinrichs, A.; Baumann, K.; Banz-Jansen, C.; Rody, A.; Waldmann, A.; Fischer, D. (Lübeck)

Pregnancy-Associated Breast Cancer: Clinicopathological Characteristics of 20 Cases with a Focus on Identifiable Causes of Diagnostic Delay Basaran, D.; Tugral, M.; Beksac, K.; Ozyuncu, O.; Aran, O.; Beksac, S. (Ankara)

\section{Novel Insights from Clinical Practice}

Three Metachronous Cases of Her-2-Positive Breast Cancer Accompanied with Thyroid Cancer

Gao, Q.; Zheng, Y.; Wang, B.; Wu, Z.; Ren, G. (Hangzhou) 